\title{
Periosteosynthetische Fraktur - die Fraktur im Bereich implantierter Osteosynthesematerialien
}

\author{
Thomas Mückley
}

\section{Zusammenfassung}

Implantatassoziierte Frakturen, die während oder nach einer Osteosynthese auftreten, werden im klinischen Alltag häufig den periimplantären Frakturen gleichgestellt, besser aber als periosteosynthetische Frakturen bezeichnet. Periosteosynthetische Frakturen sind deutlich seltener als periprothetische Frakturen, nehmen aber im klinischen Alltag merklich zu. Sie werden definiert als Frakturen im Übergangsbereich vom durch eine Osteosynthese versteiften Knochen zum gesunden Knochen. Die Unterteilung zwischen intra- und postoperativ aufgetretenen periosteosynthetischen Frakturen ist sinnvoll. In der Literatur sind außer Einzelfallbeschreibungen keine Publikationen zu finden, die sich mit dieser Problematik befassen. Die Therapie von periosteosynthetischen Frakturen ist schlecht standardisierbar und benötigt eine hohe unfallchirurgisch-orthopädische Expertise.

\section{Periosteosynthetic Fracture - Fractures in the Region of Osteosynthesis Implants}

In the clinical setting implant-associated fractures are often called periimplant fractures, but better referred as periosteosynthetic fractures because they occur during or after osteosynthesis. Periosteosynthetic fractures are much less common than periprosthetic fractures, but incidence is growing. They are defined as fractures in the transition region from the stiffened bone by a osteosynthesis to the healthy bone. The distinction between intra- and postoperative periosteosynthetic fractures is helpful. In the literature, only case reports deal with this problem. The treatment of periosteosynthetic fractures is poorly standardized and requires high orthopedic surgical expertise.

\section{Einleitung}

Die größte Gruppe der implantatassoziierten Frakturen bilden die periprothetischen Frakturen. Frakturen im Bereich von implantierten Hüftgelenk-, Kniegelenk-, Schultergelenk- und Ellenbogengelenkprothesen sind aufgrund der entsprechend hohen Primärimplantationen, der zunehmenden Anzahl älterer Personen in unserer Bevölkerung und der gleichzeitig gestiegenen Aktivität dieser Personen mit zunehmender Inzidenz zu erwarten und stellen per se eine unfallchirurgisch-orthopädische Herausforderung dar.

Neben den periprothetischen Frakturen gibt es aber implantatassoziierte Frakturen, die nach oder während einer Osteosynthese auftreten. Diese Gruppe von Frakturen wird im klinischen Alltag häufig den periimplantären Frakturen gleichgestellt, besser aber als periosteosynthetische Frakturen bezeichnet. Periosteosynthetische Frakturen sind deut- lich seltener als periprothetische Frakturen, nehmen aber aufgrund der immer häufiger werdenden Alterstraumatologie im klinischen Alltag merklich zu.

Die Inzidenz periosteosynthetischer Frakturen nimmt aufgrund der immer häufiger werdenden Alterstraumatologie zu.

Periosteosynthetische Frakturen werden definiert als Frakturen im Übergangsbereich vom durch eine Osteosynthese versteiften Knochen zum gesunden Knochen. Sie werden damit von anderen Komplikationsmöglichkeiten nach Frakturversorgung, wie u.a. Pseudarthrosenbildung ggf. in Kombination mit Implantatversagen, das Perforieren winkelstabiler Schrauben in angrenzende Gelenke, wie z.B. bei proximalen Humerusplatten und Cut-out von Schenkelhalsschrauben, abgegrenzt, welche i.d.R. durch kein erneutes Trauma verursacht sind.

In der Literatur sind außer Einzelfallbeschreibungen keine Publikationen zu finden, die sich mit dieser Problematik befassen. Es gibt keine Inzidenzangaben, keine anerkannten Klassifikationssysteme und die Behandlung ist sehr einzelfallorientiert. Insofern ist die Therapie von periosteosynthetischen Frakturen schlecht standardisierbar und benötigt eine hohe unfallchirurgisch-orthopädische Expertise.
Tab. 1 Ursachenanalyse der intraoperativen periosteosynthetischen Fraktur.
Nagelosteosynthese

Fixateur-externe- und

Schraubenosteosynthese

osteosyntheseunabhängige

Risikofaktoren u. a. falscher Eintrittspunkt, unzureichende Eintrittspunktpräparation, geringe Kortex-Knochenkanal-Ratio, geringe Knochenkanal-Marknageldurchmesser-Ratio

Fehlbohrungen auf gleicher Höhe z. B. kombiniert mit Repositionsmanövern

Osteoporose, verminderte Knochenqualität, Knochendefekte, Knochendeformitäten 

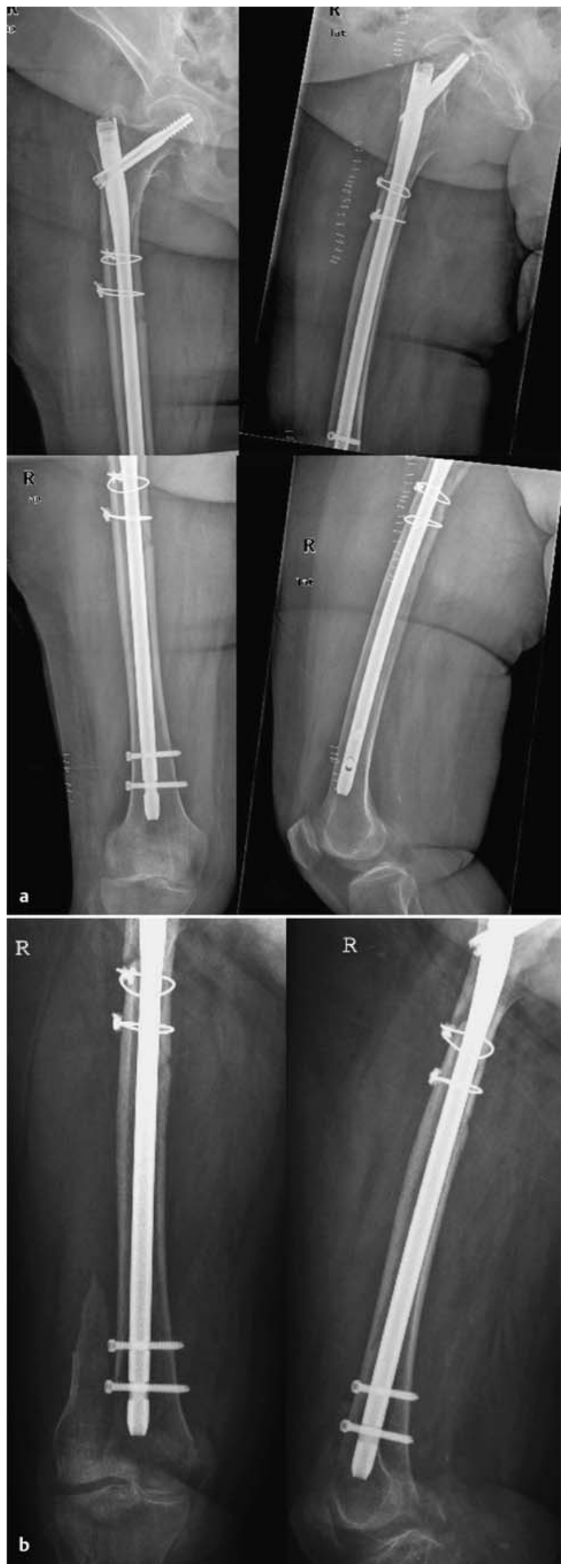

Abb. 1 a und $b$

a Subtrochantäre

Femurfraktur mit langem proximalen Femurmarknagel versorgt. Distal geringe Kortex-Knochenkanal-Ratio und ventrale Lage des Nagels. b Postoperative periosteosynthetische distale Femurfraktur bei erneutem Sturz.

\section{Hauptteil}

Neben der Unterteilung zwischen periprothetischen und periosteosynthetischen Frakturen ist die Trennung zwischen intra- und postoperativ aufgetretenen Frakturen sinnvoll.

Die Trennung zwischen intra- und postoperativ aufgetretenen periosteosynthetischen Frakturen ist sinnvoll.

In der Hüftendoprothetik wird die Inzidenz intraoperativer Frakturen bei Primäreingriffen mit ca. $1 \%$ angegeben, während sie bei Hüftprothesenwechseln auf 3-28\% steigt [1,3]. Als Risikofaktoren gelten Osteoporose, eine verminderte Knochenqualität und Deformitäten des proximalen Femurs; in der Revisionsendoprothetik zudem Knochendefekte, ein zu weites Aufraspeln des Markraums, eine geringe Kortex-Knochenkanal-Ratio, Osteolysen und die Verwendung von Revisionsschäften mit großen Durchmessern und langem geraden Design $[6,8]$.

Postoperative Frakturen in der Endoprothetik haben eine Inzidenz von ca. 0,4$1,7 \%$ bei Primärprothesen und $4 \%$ bei Revisionsprothesen $[1,5]$. Hier zeigen sich weitere Risikofaktoren wie Stress Riser (z.B. ehemalige Schraubenlöcher im Bereich der Prothesenspitze, angrenzende Implantate) und gelockerte Implantate. Das durchschnittliche Zeitintervall zwischen primärer TEP und Fraktur beträgt nach Lindahl et al. 7,4 Jahre, bei Revisionsprothesen beträgt das Zeitintervall im Mittel 3,9 Jahre [5]. Als Ursache wird mehrheitlich der Sturz in der Ebene angegeben, zudem zeigen Lindahl et al. auf, dass die Inzidenz von Frakturen ohne Trauma in der Revisionsendoprothesengruppe bis auf $37 \%$ steigt [5]. Entsprechende Inzidenz- und Risikoangaben liegen für periosteosynthetische Frakturen nicht vor. Bezüglich der Ursachenanalyse ist es hilfreich, intraoperative von postoperativen periosteosynthetischen Frakturen zu trennen. Die intraoperativen periosteosynthetischen Frakturen sind überwiegend bei Marknagelosteosynthesen zu finden (Tab. 1).

Die intraoperativen periosteosynthetischen Frakturen sind überwiegend bei Marknagelosteosynthesen zu finden.

Mögliche Risiken können ein fehlerhafter Nageleintrittspunkt bzw. eine unzureichende Präparation des Nageleintrittspunkts, eine geringe KortexKnochenkanal-Ratio (Abb. 1) sowie eine 


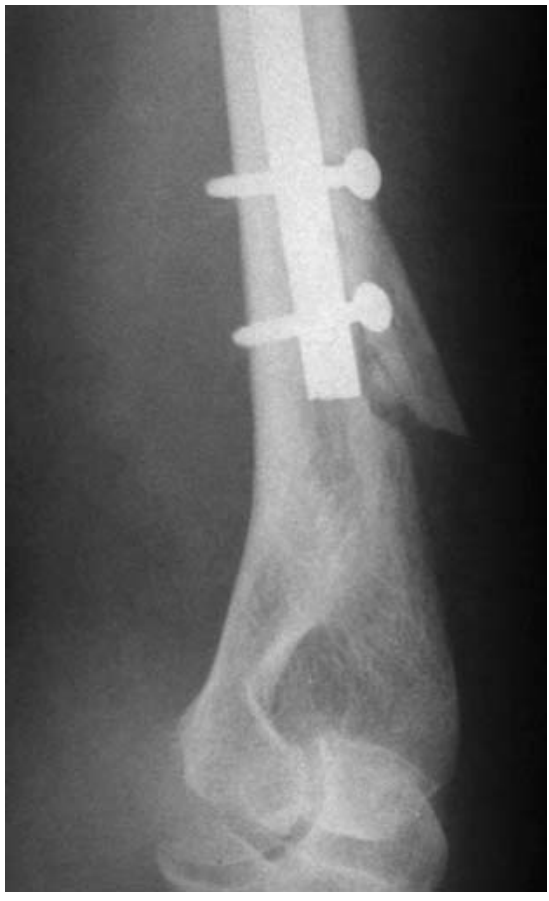

Abb. 2 Intraoperative periosteosynthetische Fraktur im Rahmen einer retrograder Humerusnagelung bei unzureichender Präparation des Nageleintrittportals.

geringe Knochenkanal-Marknageldurchmesser-Ratio sein. Letztere kann durch die Variation des Marknageldurchmessers und die Präparation des Markkanals, z.B. mit flexiblen Bohrwellen, beeinflusst werden. Klassische Beispiele für die intraoperativen periosteosynthetischen Frakturen im Rahmen von Marknagelungen sind Frakturen im Bereich des Eröffnungsportals für die retrograde Humerusnagelung (Abb.2), die Frakturen im Bereich der Nagelspitze bei der antegraden bzw. retrograden Femurnagelung mit langen, die ganze Diaphyse überbrückenden Marknägeln (Abb.3), sowie Frakturen im Rahmen von proximalen Femurmarknagelungen. Die intraoperativen Frakturen im Rahmen von proximalen Femurmarknagelungen sind implantatdesign- und erfahrungsabhängig und werden in großen Serien mit $0,5 \%$ angegeben [2]. Bei Plattenosteosynthesen sind intraoperative periosteosynthetische Frakturen die absolute Ausnahme. Bei Schrauben- und Fixateur-externe-Osteosynthesen können sie i.d.R. nur nach mehrfachen Fehlbohrungen auf gleicher Höhe mit entsprechender Schwächung der Kortikalis und dann meist im Rahmen von Repositionsmanövern auftreten.

Postoperative periosteosynthetische Frakturen können prinzipiell jede Stelle des nicht durch die Osteosynthese stabi-

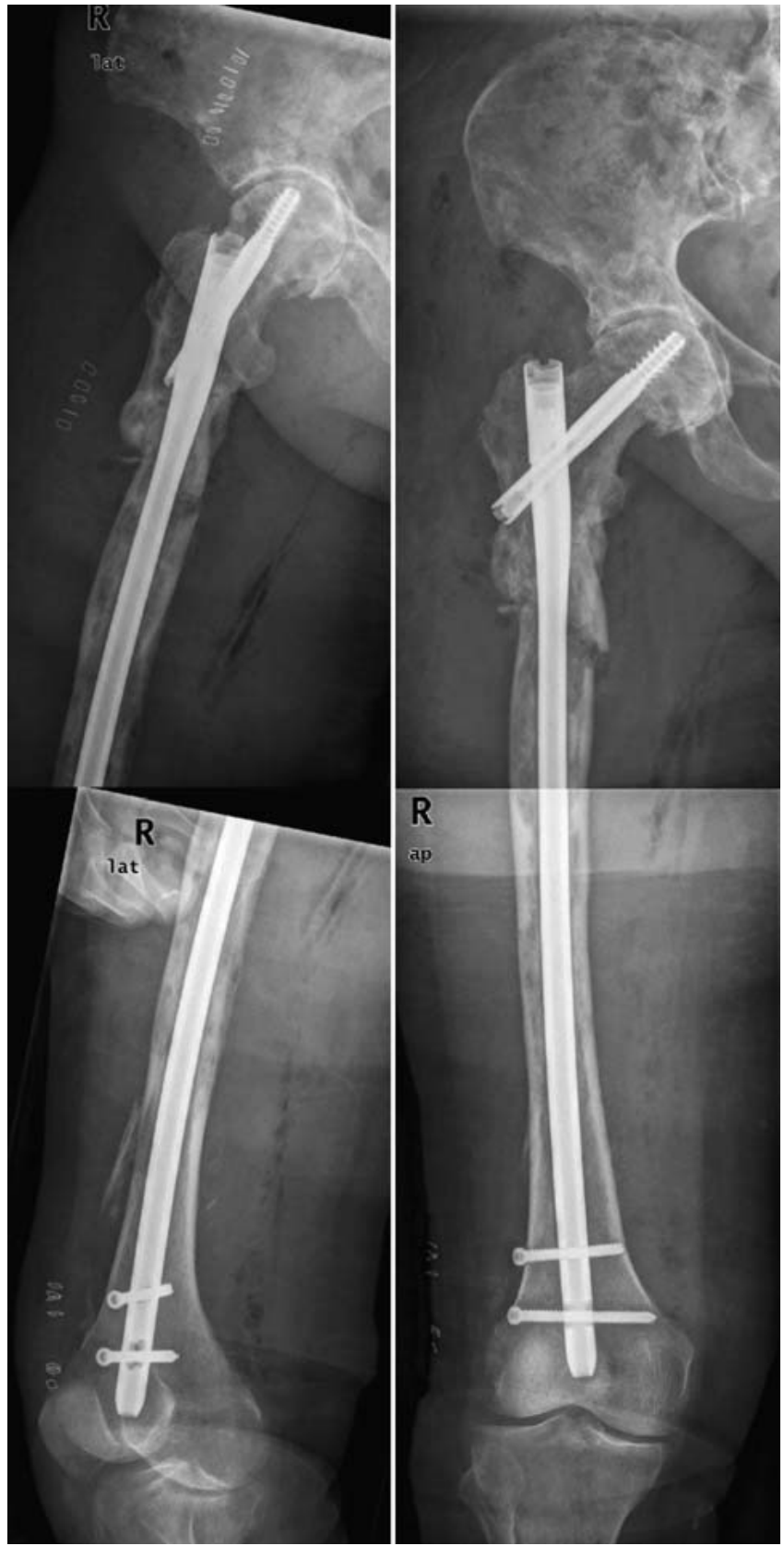

Abb. 3 Intraoperative periosteosynthetische Fraktur im Rahmen einer langen Gammanagelung im Bereich des distalen Femurs.

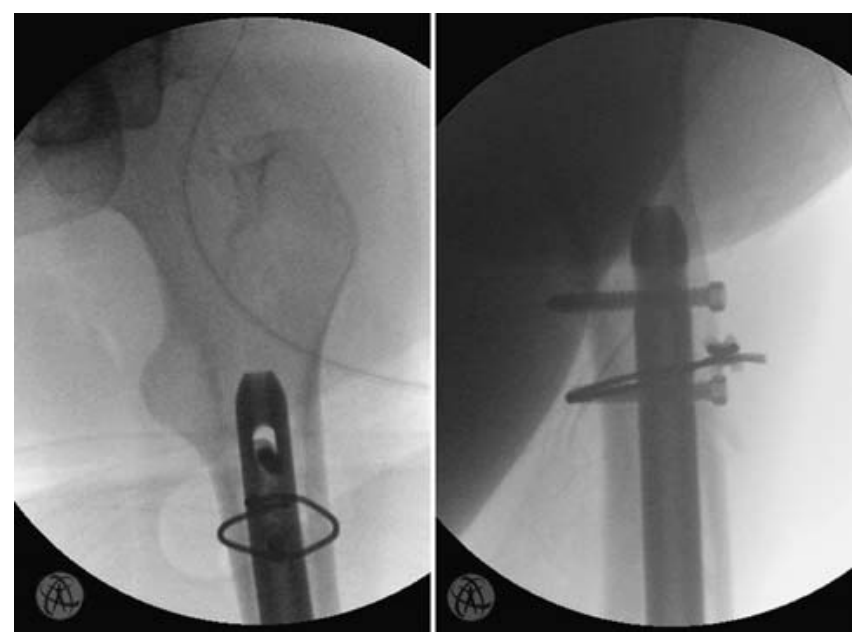

Abb. 4 Intraoperative periosteosynthetische Fraktur im Rahmen einer retrograden Femurnagelung im Bereich der Nagelspitze. Bei unikortikalem Defekt Stabilisierung mit additiver Kabelcerclage. 
Tab. 2 Ursachenanalyse der postoperativen periosteosynthetischen Fraktur.

\begin{tabular}{ll}
$\begin{array}{l}\text { osteosyntheseabhängige } \\
\text { Risikofaktoren }\end{array}$ & $\begin{array}{l}\text { knöcherne Schwachstellen wie z. B. Bohrungen für Verrie- } \\
\text { gelungsschrauben am Nagelende, Bohrungen für Schrau- } \\
\text { ben am Plattenende, ehemalige Fixateur externe-Bohrun- } \\
\text { gen, subtrochantäre Bohrungen für Schenkelhalsschrauben }\end{array}$ \\
\hline $\begin{array}{l}\text { osteosyntheseunabhängige } \\
\text { Risikofaktoren }\end{array}$ & $\begin{array}{l}\text { u. a. erhöhte Sturzneigung, Osteoporose, verminderte } \\
\text { Knochenqualität, Knochendefekte, Knochendeformitäten }\end{array}$
\end{tabular}

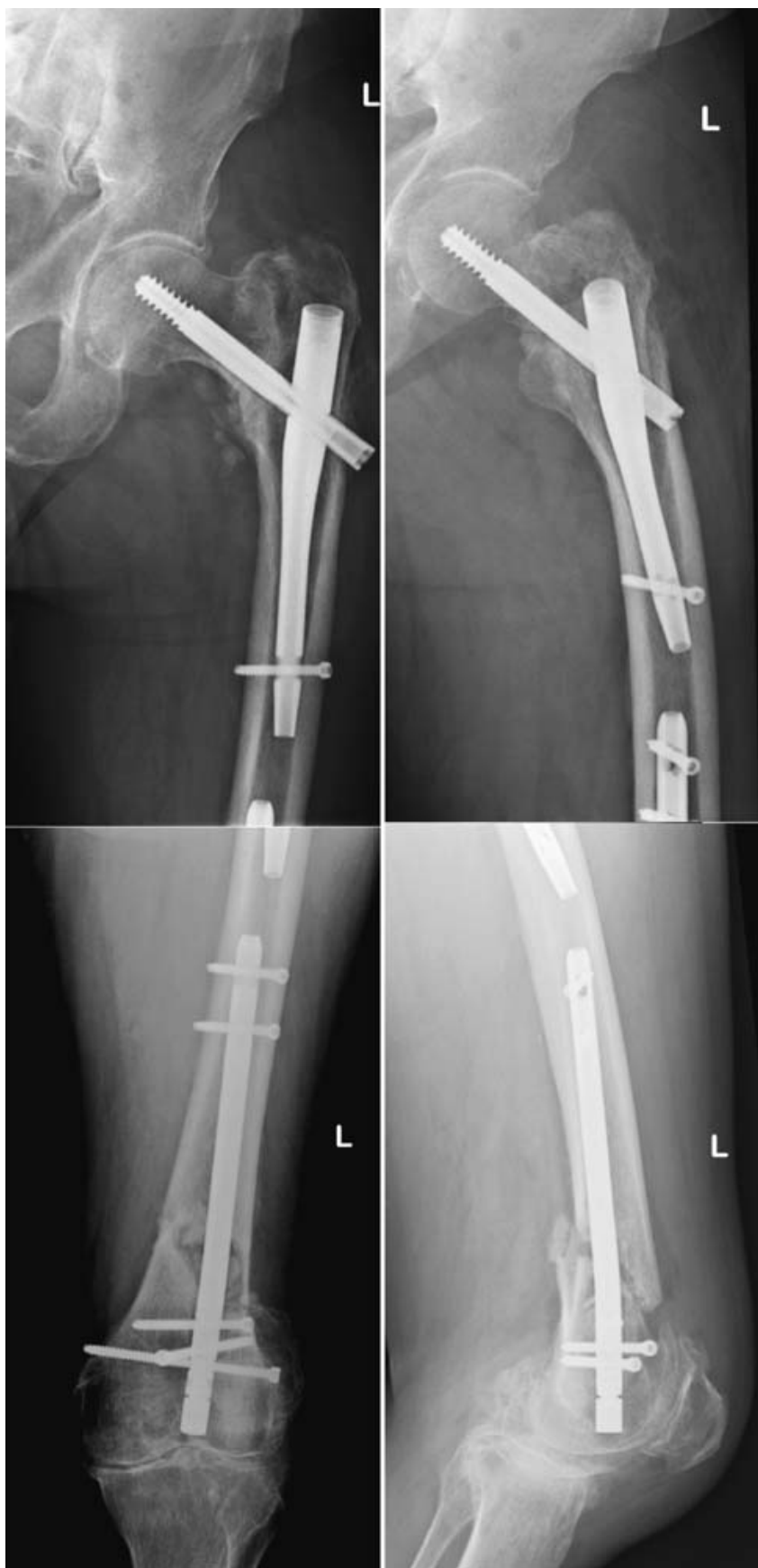

Abb. 5 Vermieden werden sollten sog. „kissing implants“ ohne Überbrückung der Implantate und mit nur geringer implantatfreier Knochenstrecke.
Prädilektionsstellen für postoperative periosteosynthetische Frakturen sind zum einen knöcherne Schwachstellen wie z.B. Bohrungen für Verriegelungsschrauben am Nagelende, Bohrungen für die am Plattenende positionierten Schrauben einer Plattenostesynthese, ehemalige Fixateur-externe-Bohrungen und subtrochantäre Bohrungen für Schenkelhalsverschraubungen (Tab.2). Von diesen knöchernen Schwachstellen ziehen die Frakturen dann meist in den nicht durch die Osteosynthese überbrückten Knochenbereich. Diese Form der postoperativen periosteosynthetischen Fraktur ist noch komplexer in der Behandlung als die Frakturformen, die ausschließlich den nicht vom Osteosyntheseimplantat überbrückten Knochenbereich betreffen, da immer die Stabilität der Primärosteosynthese infrage gestellt werden muss.

Bei den postoperativen periosteosynthetischen Frakturen muss immer die Stabilität der Primärosteosynthese geprüft und infrage gestellt werden.

Ggf. muss die neue, geplante Osteosynthese über die primäre, nun instabile Osteosynthese und die periosteosynthetische Fraktur ausgedehnt werden. Ist die primäre Ostesynthese prinzipiell noch stabil, kann im besten Fall die sekundäre Fraktur mit einer 2. Osteosynthese isoliert adressiert werden. Als Risikofaktoren gelten, neben einer erhöhten Sturzneigung, Osteoporose, verminderte Knochenqualität, Deformitäten des Knochens und Knochendefekte, sowie Stress Riser (z.B. Bohrlöcher im Bereich des Plattenendes bzw. der Nagelspitze). Als Ursachen sind mehrheitlich erneute Stürze mit Beteiligung der unteren und/ oder oberen Extremität festzustellen. Die Inzidenz von periosteosynthetischen Frakturen ohne Trauma ist nicht publiziert, aber sicherlich vergleichbar mit den Daten für die periprothetischen Frakturen [5].

\section{Diagnostik}

Grundsätzlich ist im Rahmen der allgemeinen Untersuchung und Anamneseerhebung eine Einschätzung der Funktion der Extremität vor dem Unfallereignis notwendig - ähnlich der Einschätzung in „happy hip“ oder „unhappy hip“ bei einer periprothetischen Fraktur nach Hüftendoprothese [7]. Eine entsprechende klinische Symptomatik kann Hinweise auf eine bereits vor dem erneuten Unfallereignis bestehende Im-
Postoperative periosteosynthetische Frakturen können prinzipiell jede Stelle des nicht durch die Osteosynthese stabilisierten Knochens betreffen, beginnen jedoch häufig im Bereich des einliegenden Osteosynthesematerials. jedoch häufig im Bereich des einliegenden Osteosynthesematerials und können gleichzeitig auch zu einem akuten Versagen der Osteosynthese führen (z.B. Ausriss der Platte). 
plantatproblematik (Lockerung etc.) geben. Anamnestisch ist des Weiteren das Unfallereignis von besonderer Bedeutung. Es ist zu klären, ob ein adäquates Unfallereignis vorlag, das die Entstehung der Fraktur rechtfertigen kann. Ansonsten sind die diversen Ursachen einer reduzierten Knochenqualität abzuklären (Osteoporose, Osteolysen etc.). Eine Gefäß- oder Nervenverletzung muss durch die klinische Untersuchung ausgeschlossen werden. Begleitende Weichteilverletzungen sind entsprechend $\mathrm{zu}$ erheben, zu klassifizieren und zu therapieren.

Die Diagnose einer periosteosynthetischen Fraktur wird im Regelfall projektionsradiografisch gestellt. Dabei ist auf die Mitabbildung der angrenzenden Gelenke zu achten.

Zusätzlich zur nativen Röntgendiagnostik wird in den meisten Fällen die Durchführung einer Computertomografie (CT) angestrebt. Sie dient im Wesentlichen der detaillierten Frakturdarstellung, insbesondere der Beurteilung einer Instabilität der Osteosynthese, Analyse von Knochendefekten, der Darstellung undislozierter Frakturausläufer sowie möglicher Frakturausläufer in die angrenzenden Gelenke. Zudem kann hiermit die umgebende Knochenqualität und die primäre Fraktur hinsichtlich der knöchernen Durchbauung beurteilt werden. Die Informationen der CT-Untersuchung helfen bei der Beantwortung folgender Fragen:

1. Ist die primäre Fraktur evtl. bereits durchbaut?

2. Ist die einliegende Osteosynthese gelockert?

3. Ist eine Reosteosynthese möglich?

4. Welches Implantat bietet sich hierfür an?

Die CT-Untersuchung dient der detaillierten Frakturdarstellung.

Bei projektionsradiografischem oder anamnestischem Verdacht einer pathologischen Fraktur ist die CT-Untersuchung obligat.

Besteht klinisch oder projektionsradiografisch der Verdacht einer osteoporotischen Genese der Fraktur, sollte eine Knochendichtebestimmung durchgeführt werden. Neben der Einleitung einer systemischen Osteoporosetherapie stellt die Osteodensitometrie einen wichtigen Aspekt einer möglichen Indikation für ein zementaugmentiertes Osteosyntheseverfahren dar [4].

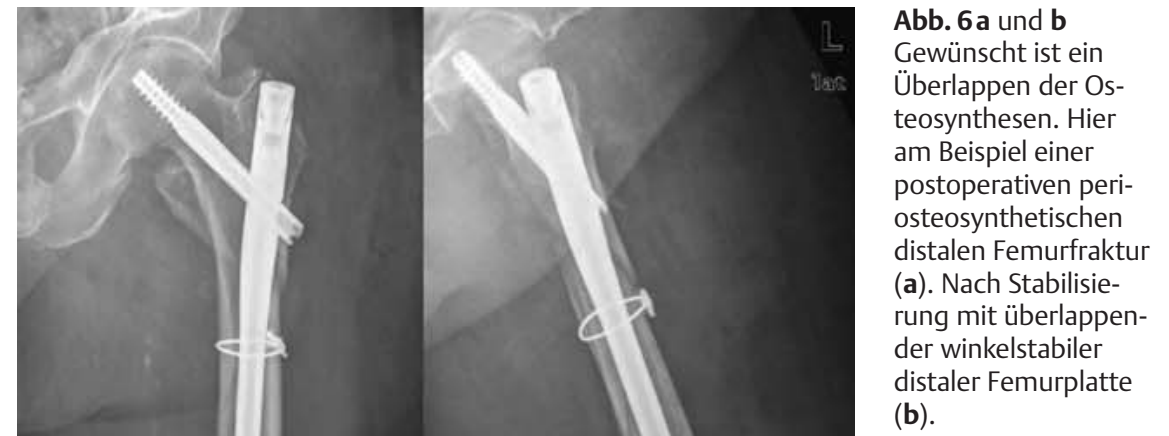

Gewünscht ist ein Überlappen der Osnthesen. Hier postoperativen peristeosynthetischen Femurfraktur rung mit überlappen(b)

Thomas Mückley: Periosteosynthetische Fraktur - die Fraktur im Bereich implantierter Osteosynthesematerialien 


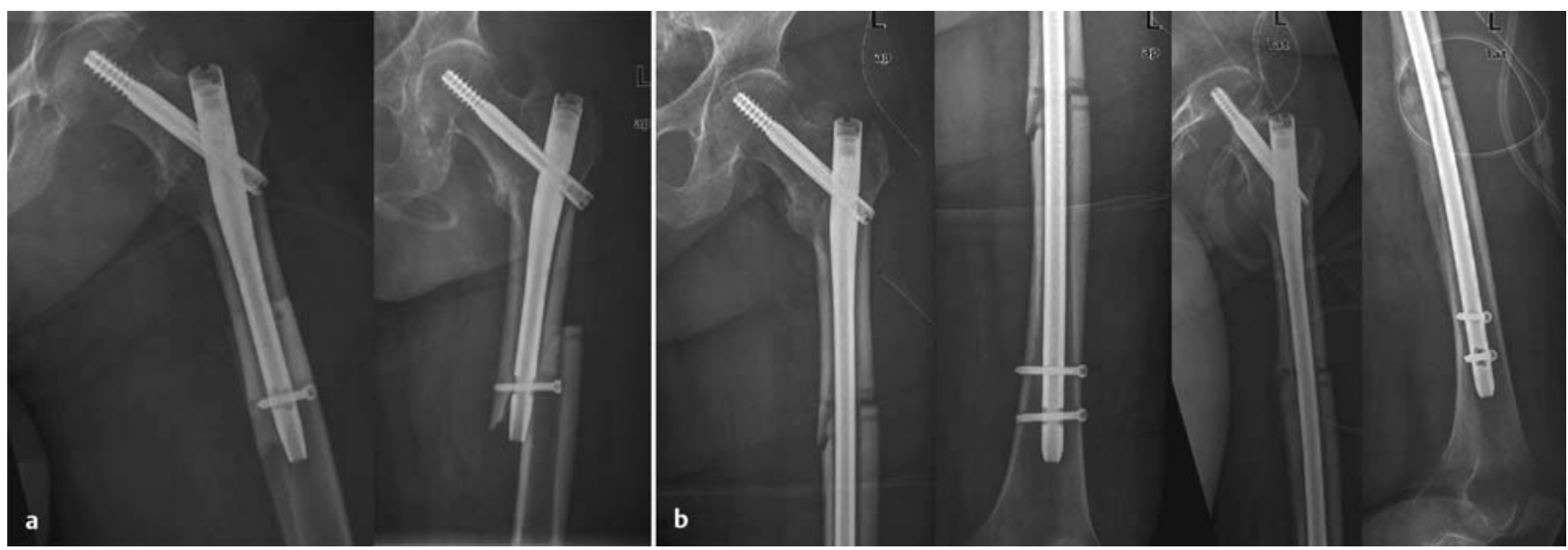

Abb. 7 a und b Postoperative periosteosynthetische Fraktur am distalen Nagelende eines proximalen Femurnagels nach Fehlbohrung (a). Nagelwechsel auf lange Nagelversion mit Überbrückung beider Frakturen (b).

\section{Therapie}

Periosteosynthetische Frakturen werden überwiegend operativ therapiert.

Einzelfälle der konservativen Therapie können intra- und postoperative periosteosynthetische Frakturen an der oberen Extremität sein, welche prinzipiell besser konservativ zu behandeln sind als Frakturen an der mehr belasteten, unteren Extremität. Da die periosteosynthetischen Frakturen überwiegend Patienten im fortgeschrittenen Lebensalter betreffen, sind die rasche Mobilisation und die regelmäßig nicht mögliche Entlastung bzw. Teilbelastung der Extremität in der Therapiewahl zu berücksichtigen. Entsprechende Dringlichkeiten in der Versorgung (OP innerhalb 24-48 Stunden nach Fraktur) von periosteosynthetischen Femurfrakturen sind wie bei der Versorgung von proximalen Femurfrakturen in der Alterstraumatologie anzunehmen.

Da intraoperative periosteosynthetische Frakturen überwiegend im Rahmen von Marknagelungen auftreten, ist die Therapie auf diese OP-Technik abzuzielen. Das sicherste Verfahren ist der Wechsel des einliegenden Marknagels auf eine längere Version mit Überbrückung der periosteosynthetischen Fraktur. Die gelingt regelhaft bei neu aufgetretenen Frakturen an der Nagelspitze kurzer Marknägel. Bei langen, die Markhöhle nach proximal und distal ausfüllenden Marknägeln sind periosteosynthetische Frakturen am Nageleintrittspunkt bzw. an der Nagelspitze schwieriger zu therapieren. Eine konservative Therapie kommt meist nicht infrage, da sie längere Ruhigstellungsphasen gelenküberbrückend erfordern würde. Dies wäre völlig konträr zu dem operativen Ansatz der Primärfraktur und wäre mit schlechten funktionellen Ergebnissen verbunden. Bei unikortikalen Defekten bzw. Fissuren können additive Kabelzerklagen zum einliegenden Marknagel eine gute Lösungsmöglichkeit sein (Abb.4). Auch additive Plattenosteosynthesen mit Überlappung der Implantate und evtl. unikortikaler Verankerung im Nagelbereich sind möglich. Sollte dies nicht möglich sein, muss der Marknagel entfernt und eine lange Plattenosteosynthese, die beide Frakturregionen stabilisiert, durchgeführt werden. Wechsel auf noch längere Marknägel bzw. Marknägel mit Gelenkkomponente sind nur in Einzelfällen möglich.

Bei postoperativen periosteosynthetischen Frakturen ist zunächst die Stabilität der einliegenden Osteosynthese abzuklären. Ist die einliegende Osteosynthese noch stabil verankert und die periosteosynthetische Fraktur weit genug entfernt, kann die Osteosynthese der periosteosynthetischen Fraktur im Idealfall isoliert durch eine 2. Osteosynthese stabilisiert werden. Hierbei sind die allgemeinen Osteosyntheseprinzipien $\mathrm{zu}$ beachten.

Vermieden werden sollten nah aneinander endende Implantate mit nur geringer freier Knochenstrecke (sog. kissing implants, Abb.5). Die geringe freie Knochenstrecke stellt durch die Stresskonzentration in diesem Bereich eine Sollbruchstelle für zukünftige Frakturen dar. Insofern ist die Überlappung der Osteosynthesestrecken wünschenswert (Abb. 6). Trotz stabiler Verankerung muss manchmal die Primärosteosynthese dennoch entfernt und auf eine Osteo- synthese mit Einschluss der primären und sekundären Fraktur gewechselt werden (Abb. 7). In Einzelfällen sind Endoprothesenimplantationen ggf. mit zusätzlicher Osteosynthese notwendig, um nicht mehr rekonstruierbare gelenknahe Frakturen bzw. Gelenkfrakturen zu adressieren.

Postoperative periosteosynthetische Frakturen können auch bei inadäquatem Trauma auftreten. Mögliche Ursachen sind u.a. ausgeprägte Osteoporose, Knochendefekte und Deformitäten. Diese sind entsprechend in der Therapie zu berücksichtigen. Neben der medikamentösen Osteoporosetherapie kommen Zementaugmentationsverfahren für die Implantate, Knochen- und Spongiosatransplantationen und Korrekturen von Deformitäten zum Einsatz. Mittlerweile sind entsprechende standardisierte Zementaugmentationsverfahren um Schenkelhalsklingen von proximalen Femurnägeln und um Verriegelungsschrauben von winkelstabilen proximalen Humerusplatten verfügbar. Weitere Anwendungsbereiche, wie z.B. distale Femurplatten und proximale Tibiaplatten, werden sicherlich bald folgen.

Auch implantatspezifische Aspekte können postoperative periosteosynthetische Frakturen ohne adäquates Trauma verursachen und müssen berücksichtigt werden. Ältere proximale Femurnägel mit 6,28-mm-Verriegelungsschrauben distal und $10^{\circ}$-Valgusangulation haben häufiger zu Verklemmung und Stresskonzentration im proximalen Femur und $\mathrm{zu}$ periosteosynthetischen Frakturen geführt als modernere, modifizierte Marknageldesigns. 
Da intraoperative periosteosynthetische Frakturen nahezu immer vermeidbar sind und auch die postoperativen periosteosynthetischen Frakturen nicht selten auf vermeidbare Fehler zurückzuführen sind, ist die Prävention von besonderer Bedeutung.

Der Prävention von periosteosynthetischen Frakturen kommt eine besondere Bedeutung zu.

Dies muss in der Facharztweiterbildung und den operationstechnischen Kursen berücksichtigt werden. Zudem sind die Sturzkrankheit wie auch die Osteoporose entsprechend abzuklären und zu therapieren.

\section{Literatur}

${ }^{1}$ Berry DJ. Epidemiology: hip and knee. Orthop Clin North Am 1999; 30: 183-190

2 Kukla C, Heinz T, Gaebler et al. The standard gamma nail: a critical analysis of 1000 Cases. J Trauma 2001; 51: 77-83

3 Kavanagh, BF, Wallrichs S, Dewitz $M$ et al. Charnley low-friction arthroplasty of the hip. Twenty-year results with cement. J Arthroplasty 1994; 9: 229-234

${ }^{4}$ Klos K, Wähnert D, Gueorguiev B et al. Development of a technique for cement augmentation of nailed tibiotalocalcaneal arthrodesis constructs. Clin Biomech 2010; 25: 576-581

${ }^{5}$ Lindahl H, Malchau H, Herberts P et al. Periprosthetic femoral fractures classification and demographics of 1049 periprosthetic femoral fractures from the Swedish National Hip Arthroplasty Register. J Arthroplasty 2005; 20: 857-865

${ }^{6}$ Meek RM, Garbuz DS, Masri BA et al. Intraoperative fracture of the femur in revision total hip arthroplasty with a diaphyseal fitting stem. J Bone Joint Surg Am 2004; 86: 480-485
${ }^{7}$ Ninan $T$ M, Costa ML, et al. Classification of femoral periprosthetic fractures. Injury 2007; 38: 661-668

8 Zalzal P, Gandhi R, Petruccelli D et al. Fractures at the tip of long-stem prostheses used for revision hip arthroplasty. J Arthroplasty 2003; 18: 741-745

Prof. Dr. med. habil. Thomas Mückley

Chefarzt

Klinik für Orthopädie und

Unfallchirurgie

HELIOS Klinikum Erfurt

Nordhäuser Straße 74

99089 Erfurt

Tel.: 0361/781-2360

Fax: 0361/781-2362

thomas.mueckley@helios-kliniken.de 